\title{
STATE-DEPENDENT UTILITY
}

\author{
JAIME A. LONDOÑO, ${ }^{*}$ Universidad Nacional de Colombia
}

\begin{abstract}
We propose a new approach to utilities in (state) complete markets that is consistent with state-dependent utilities. Full solutions of the optimal consumption and portfolio problem are obtained in a very general setting which includes several functional forms for utilities used in the current literature, and consider general restrictions on allowable wealths. As a secondary result, we obtain a suitable representation for straightforward numerical computations of the optimal consumption and investment strategies. In our model, utilities reflect the level of consumption satisfaction of flows of cash in future times as they are (uniquely) valued by the market when the economic agents are making their consumption and investment decisions. The theoretical framework used for the model is the one proposed in Londoño (2008). We develop the martingale methodology for the solution of the problem of optimal consumption and investment in this setting.

Keywords: State-dependent utility; stochastic flow; optimal consumption and investment

2000 Mathematics Subject Classification: Primary 91B28

Secondary $91 \mathrm{~B} 16$
\end{abstract}

\section{Introduction}

The problem of optimal consumption and investment for a 'small investor' whose actions do not influence market prices is at the core of portfolio management and it is the building block for the development of equilibrium theory. The modern treatment of this problem when asset prices follow Itô processes started with the seminal works of [39] and [40]. Using a 'martingale' approach, Cox and Huang [10] and Karatzas et al. [27] solved the problem in more general settings in the case of complete markets. A representation formula is derived in [41] in terms of the expectation of random variables which involve Malliavin derivatives of the coefficients of the model. The latter gives theoretical formulae for optimal portfolios and consumption strategies.

In order to obtain numerical representations for the structure of optimal portfolios and consumption processes, it is natural to use methods based on the dynamic-programming approach. However, numerical schemes based on partial differential equations become increasingly difficult to evaluate when the dimension of the underlying state variable increases, and even standard techniques are somehow inappropriate for the solution of the partial differential equations that arises in small dimensions (see [13]). As a result, attention has been directed to models admitting closed-form solutions (see [30], [33], and [43]), specifications which are computationally tractable based on dynamic-programming techniques (see [2]-[4], and [7]), discrete-time models based on approximated Euler equations (see [1], [5], [6], and [12]), or Monte Carlo techniques (see [11] and [15]).

Received 30 August 2007; revision received 20 December 2008.

* Postal address: Departamento de Matemáticas, Universidad Nacional de Colombia, Bogotá, Colombia.

Email address: jaime.a.londono@gmail.com 
However, the main drawback of the standard models for optimal consumption and investment is their lack of agreement with empirical data. These inconsistencies are documented with the name of several puzzles such as the 'equity premium puzzle' [37], the 'risk-free rate puzzle' [44], and the 'risk-aversion puzzle' [25]. In order to address these problems, several generalizations have been suggested. One of these proposes habit formation for consumers. Some examples are given in [9], [21], [22], and [23]. Another approach is the construction of recursive utility. Some references for this are [16], [17], and [32]. A different way to account for the discrepancy between theory and empirical data is the assumption of transaction costs for changes in consumption levels. Some references for this approach are [14], [36], and [42], to cite a few.

In fact, one of the reasons why standard utility models fail to fit economic behavior might be the fact that state-independent utilities are not appropriate for modeling the behavior of human beings. For instance, see [28] and [29]. Partly motivated by the above, some literature in finance has focused on state-dependent utilities to explain the behavior of individual consumers and investors. Some recent references are [8], [18], [19], and [38], among others. For instance, Melino and Yang [38] generalized the model of [17] by allowing preferences where the elasticity of inter-temporal substitution and coefficient of risk aversion are state dependent. In a MehraPrescott economy, they were able to match the US historical first two moments of the returns on equity and the risk-free rate. Similarly, Gordon and St-Amour [18], [19], using expected-utility preferences, were able to find a good match to post World War II data.

In this paper we propose a new approach for utilities in state-complete markets. See [34] and [35] for the definition of a state-complete market. Mathematically, this model looks similar to the standard model for utilities, but its interpretation is consistent with a model with statedependent utilities. The traditional approach is to consider that utilities reflect the level of 'happiness' for consumption levels in the future (discounted by the value of money in a bank account). See [26]. However, our guiding principle in this paper is different: we believe that agents have utilities for consumption of flows of money in future times as they are valued (by the market) at the time when they are making their consumption and investment decisions. Another way to look at this is that people tend to value things according to their social and economic context, instead of just looking at quantitative values. For instance, people tend to appreciate more the ability to have enough money to pay off their debts in depression times than the ability to buy luxuries in good times. In fact, state dependence is consistent with recent evidence from experimental psychology, where good times bring a positive mood for investors and a heightened pain from any potential loss (see [24, pp. 417-435] and the references therein). A detailed account that explains why state-dependent utilities have the potential to resolve the equity premium puzzle is discussed in [38]. In a Mehra-Prescott economy, they showed that in order to match data on the first two moments of asset returns, a stochastic discount factor is necessary that is very sensitive to the current state, and that it should be consistent with a counter-cyclical pattern of risk aversion. However, with iso-elastic expected utility preferences, the stochastic discount factor varies over time with the rate of consumption growth only being realized in the next period.

The above remarks completely change the optimization problem we consider, with the advantage that most of the tools used to solve the old problem can be used in this setting. In particular, the martingale methodology is available. A consequence of adopting this approach is that full solutions of the optimal consumption and portfolio problem are obtained in a very general setting that includes several of the functional forms for utilities in the literature, and considers quite general restrictions on allowable wealths. As a secondary result, we obtain 
suitable representation for straightforward numerical computations of the optimal consumption and investment strategies.

The theoretical framework used to solve the above problem is the one proposed in [35]. Above, we named the processes described in the cited paper as consistent measurable processes; these are processes whose evolution between any two times depends only on the evolution of the underlying Brownian motion and satisfies some consistency conditions. The methodology described can be used in processes that are a generalization of Brownian flows [31], and is applicable to those processes that are the solutions of classical Itô stochastic differential equations, even when the volatilities and drifts are just locally $\delta$-Hölder continuous for some $\delta>0$. It can also be straightforwardly adapted to stochastic volatility models whose evolution of the underlying drifts and volatilities are described by classical stochastic Itô differential equations. As discussed in [35], the theoretical framework is potentially useful when the underlying randomness is generated by a (not necessarily continuous) Lévy process. At the same time, the theoretical framework described in [35] is a particular case of the theory of arbitrage and valuation presented in [34]. To the best of the author's knowledge, this theory of arbitrage and valuation is the most general existing theory in the case of (continuous) semimartingales driven by Brownian filtrations with continuous coefficients.

The paper is organized as follows. In Section 2 we review the model and definitions presented in [35], and review the definitions of utility that we use in this paper. In Section 3 we present a martingale methodology needed to address the cited problem for the model described in [35], which plays the role of the martingale methodology of [10], [27], and [41] in the current context. Finally, in Section 4 we present the main results on optimal consumption and investment.

\section{The model}

First we introduce some notation which will be frequently used in this paper. Let $\mathbb{D} \subset \mathbb{R}^{k}$ be an open connected set. Let $m$ be a nonnegative integer. We denote by $C^{m, \delta}\left(\mathbb{D}: \mathbb{R}^{n}\right)$ the Fréchet space of $m$-times continuously differentiable functions whose $m$ th-order derivatives are $\delta$-Hölder continuous with semi-norms $\|f\|_{m, \delta: K}$ defined in [31, Section 3.1], where $K \subset \mathbb{D}$ is a compact set and $0 \leq \delta \leq 1$. In the case in which $m=0$ or $\delta=0$ we denote $C^{m, \delta}\left(\mathbb{D}: \mathbb{R}^{n}\right)$ simply by $C^{\delta}\left(\mathbb{D}: \mathbb{R}^{n}\right)$ or, respectively, $C^{m}\left(\mathbb{D}: \mathbb{R}^{n}\right)$.

We consider a $d$-dimensional Brownian motion $\left\{W(t), \mathscr{F}_{t} ; 0 \leq t \leq T\right\}$ starting at 0 and defined on a complete probability space $(\Omega, \mathcal{F}, \mathrm{P})$, where $\mathcal{F}=\mathscr{F}_{T}$ and $\left\{\mathcal{F}_{t}, 0 \leq t \leq T\right\}$ is the $\mathrm{P}$ augmentation by the null sets of the natural filtration $\mathcal{F}_{t}^{W}=\sigma(W(s), 0 \leq s \leq t)$. Let $\left(\mathcal{F}_{s, t}\right)=\left\{\mathcal{F}_{s, t}, 0 \leq s \leq t \leq T\right\}$ be the two-parameter filtration, where $\mathcal{F}_{s, t}$ is the smallest sub$\sigma$-field containing all the null sets and $\sigma\left(W_{s}(u) \mid s \leq u \leq t\right)$, where $W_{s}(u) \equiv W(u)-W(s)$. For each $0 \leq s \leq T$, we also define the $\sigma$-field $\mathcal{P}_{s}$ of progressively measurable sets after time $s$ as the $\sigma$-field of sets $P \in \mathscr{B}([s, T]) \otimes \mathcal{F}_{s, T}$, the product $\sigma$-field, such that $\chi_{P}(t, \omega), t \geq s$, is an $\mathcal{F}_{s, t}$ progressively measurable (in $t$ ) process, where $\chi$ is the indicator function. We denote by $\mu_{s}$ the measure on $\mathcal{P}_{S}$ defined by $\mu_{S}(P)=\mathrm{E}\left[\int_{S}^{T} \chi_{P}(t, \omega) \mathrm{d} t\right]$.

We consider $n+1$ stocks whose evolution price process $P$ is a consistent semimartingale of class $C\left(\mathbb{R}_{+}^{n+1}: \mathbb{R}_{+}^{n+1}\right)$. A detailed account of consistent processes, financial markets, wealth evolution structure, and other concepts used in this section can be found in [35]. For $0 \leq$ $i \leq n$, we define the price-per-share process for the ith-stock, $P_{i}$, to be the $P$-consistent semimartingale process of class $C\left(\mathbb{R}_{+}^{n+1}: \mathbb{R}_{+}\right), P_{i}=\left\{P_{i}(s, t, p)=\pi_{i} \circ P(s, t, p), p \in\right.$ $\left.\mathbb{R}^{n+1}, 0 \leq s \leq t \leq T\right\}$, where $\pi_{i}$ denotes the projection on the $i$ th-component and ' $\circ$ ' denotes the standard composition of functions. We consider $P$-consistent semimartingale processes $\sigma_{i, j}, b_{i}, \delta_{i}, r$, and $\theta_{i}$ of class $C^{\delta}\left(\mathbb{R}_{+}^{n+1}: \mathbb{R}\right)$ for some $\delta>0$, where $\mathbb{R}_{+}$denotes the set of real 
positive numbers. It is assumed that $\sigma_{i, j}, b_{i}, \delta_{i}, r$, and $\theta_{i}$ relate to $P$ through the following stochastic differential equations:

$$
\begin{gathered}
\mathrm{d} P_{i}(s, t, p)=P_{i}(s, t, p)\left(b_{i}(s, t, p) \mathrm{d} t+\sum_{1 \leq j \leq d} \sigma_{i j}(s, t, p) \mathrm{d} W_{s}^{j}(t)\right), \\
P_{i}(s, s, p)=p_{i}, \quad i=1, \ldots, n,
\end{gathered}
$$

where $W_{s}^{j}(t)=W^{j}(t)-W^{j}(s)$, and

$$
\begin{gathered}
\mathrm{d} P_{0}(s, t, p)=P_{0}(s, t, p)\left(\left(r(s, t, p)+\|\theta(s, t, p)\|^{2}\right) \mathrm{d} t+\sum_{1 \leq j \leq d} \theta_{j}(s, t, p) \mathrm{d} W_{s}^{j}(t)\right), \\
P_{0}(s, s, p)=p_{0},
\end{gathered}
$$

where $\|\cdot\|$ denotes the Euclidean norm.

Throughout this paper, we will assume that $\theta(s, t, p) \in \operatorname{ker}^{\perp}(\sigma(s, t, p))$ for all $t$ (where $\operatorname{ker}^{\perp}(\sigma(s, t, p))$ denotes the orthogonal complement of the kernel of $\left.\sigma(s, t, p)\right)$ and that

$$
b(s, t, p)+\delta(s, t, p)-r(s, t, p) \mathbf{1}_{n}=\sigma(s, t, p) \theta(s, t, p)
$$

$\mu_{s}$-almost everywhere for all $p \in \mathbb{R}_{+}^{n+1}$ and $0 \leq s \leq T$, where $\mathbf{1}_{n}^{\top}=(1, \ldots, 1) \in \mathbb{R}^{n}$. This latter assumption is equivalent to the nonexistence of state-tame arbitrage opportunities (see [34]). We point out that (1) is different to the similar equation in [35]. In fact, this choice is made because in state-complete markets $P_{0}$ would be a hedgeable financial instrument (see [34]).

The process of bounded variation $B=\{B(s, t, p)\}$, whose evolution $B(s, \cdot, p), p \in \mathbb{R}_{+}^{n+1}$, $0 \leq s \leq T$, is given by the solution of the stochastic differential equation

$$
\mathrm{d} B(s, t, p)=B(s, t, p) r(s, t, p) \mathrm{d} t, \quad B(s, s, p)=1, \quad \text { for } 0 \leq s \leq t \leq T,
$$

will be called the bond price process.

We will say that $\mathcal{M}=\left(P, b, \sigma, \delta, r, p^{0}\right)$ is a financial market with terminal time $T$ and initial time 0 if $b=\left(b_{1}, \ldots, b_{n}\right)$ is a vector of rate of return processes, $\sigma=\left(\sigma_{i, j}\right)$ is a matrix of volatility coefficient processes, $\delta=\left(\delta_{1}, \ldots, \delta_{n}\right)$ is a vector of dividend rate processes, $r$ is an interest rate process, and $\theta^{\top}(s, t, p)=\left(\theta_{1}(s, t), \ldots, \theta_{d}(s, t)\right)$ is the market price of risk, and $p^{0} \in \mathbb{R}_{+}^{n+1}$ is a vector of initial prices.

We define the state-price density process to be the continuous $C\left(\mathbb{R}_{+}^{n+1}: \mathbb{R}_{+}\right)$semimartingale process defined by

$$
H(s, t, p)=B^{-1}(s, t, p) Z(s, t, p) \text { for } p \in \mathbb{R}_{+}^{n+1}, 0 \leq s \leq t \leq T,
$$

where

$$
Z(s, t, p)=\exp \left\{-\int_{s}^{t} \theta^{\top}(s, u, p) \mathrm{d} W_{s}(u)-\frac{1}{2} \int_{s}^{t}\|\theta(s, u, p)\|^{2} \mathrm{~d} u\right\}
$$

for $0 \leq s \leq t \leq T$ and $B^{-1}(s, t, p)=1 / B(s, t, p)$.

In this paper we assume that $\sigma(s, t, p)$ is a matrix of maximal rank. In this case the financial market $\mathcal{M}$ is state complete (see [34] and [35]). It follows that if there exists an 'equivalent martingale measure'; this measure is unique (see [20]) and $H(0, t, p)$ is the unique state-price density process.

Next we discuss the concept of utility that we will use in this paper. 
Definition 1. Consider a function $U:(0, \infty) \rightarrow \mathbb{R}$ that is continuous, strictly increasing, strictly concave, and continuously differentiable, with $U^{\prime}(\infty)=\lim _{x \rightarrow \infty} U^{\prime}(x)=0$ and $U^{\prime}\left(0^{+}\right):=\lim _{x \downarrow 0} U^{\prime}(x)=\infty$. Such a function will be called a utility function.

Classic examples of utility functions are $U_{\alpha}(x)=x^{\alpha} / \alpha$ for some $\alpha \in(0,1)$ and $0 \leq x<\infty$, and $U(x)=\log (x)$. For every utility function $U(\cdot)$, we will denote by $I(\cdot)$ the inverse of the derivative $U^{\prime}(\cdot)$; the functions $I(\cdot)$ and $U^{\prime}(\cdot)$ are strictly decreasing and map $(0, \infty)$ onto itself with $I\left(0^{+}\right)=U^{\prime}\left(0^{+}\right)=\lim _{x \rightarrow 0^{+}} U^{\prime}(x)=\infty$ and $I(\infty)=\lim _{x \rightarrow \infty} I(x)=U^{\prime}(\infty)=0$. We extend $U$ by $U(0)=U\left(0^{+}\right)$, and we keep the same notation for this extension, hoping that it will be clear to the reader which function we are referring to. It is a well-known result that

$$
\max _{0<x<\infty}(U(x)-x y)=U(I(y))-y I(y), \quad 0<y<\infty .
$$

Definition 2. Consider a continuous function $U_{1}:[0, T] \times(0, \infty) \rightarrow \mathbb{R}$ such that $U_{1}(t, \cdot)$ is a utility function in the sense of Definition 1 for all $t \in[0, T]$. It follows that $I_{1}(t, x):=$ $\left(\partial U_{1}(t, x) / \partial x\right)^{-1}$, the inverse of the derivative of $U$, is a continuous function. Similarly, if a utility function $U_{2}:(0, \infty) \rightarrow \mathbb{R}$ is given then $I_{2}(x):=\left(\partial U_{2}(x) / \partial x\right)^{-1}$ is continuous. Let us define

$$
\mathcal{X}(t, y):=I_{2}(y)+\int_{t}^{T} I_{1}\left(t^{\prime}, y\right) \mathrm{d} t^{\prime} .
$$

We say that $U_{1}$ and $U_{2}$ define a state preference structure.

Under the conditions outlined in Definition 2, it is easy to see that $\mathcal{X}:[0, T] \times(0, \infty) \rightarrow$ $(0, \infty)$ is a continuous function with the property that, for each $t, \mathcal{X}(t, \cdot)$ maps $(0, \infty)$ onto itself, is strictly decreasing with

$$
\chi\left(t, 0^{+}\right)=\lim _{y \downarrow 0} X(t, y)=\infty, \quad \text { and } \quad X(t, \infty)=\lim _{y \rightarrow \infty} X(t, y)=0 .
$$

We extend $U_{1}$ and $U_{2}$ by defining $U_{1}(t, 0)=U\left(t, 0^{+}\right)$for all $0 \leq t \leq T$ and $U_{2}(0)=$ $U_{2}\left(0^{+}\right)$, and we keep the same notation for the extension of $U_{1}$ to $[0, T] \times[0, \infty)$ and the extension of $U_{2}$ to $[0, \infty)$. We hope that it will be clear to the reader which function we are referring to.

We point out that the $X^{-1}$ defined for each $t$ as $X^{-1}(t, \cdot)$, the inverse of $\mathcal{X}(t, \cdot)$, share the same abovementioned properties of $\mathcal{X}$. Next we discuss the meaning of the utility functions defined above. We should interpret $U_{1}(t, x)$ for $t \in[0, T]$ as the level of 'happiness' for an agent consuming $x$ units of wealth per unit of time at time $t$, as valued by the (state-complete) market at time 0 , when the agent is planning its consumption. Similarly, we should understand $U_{2}(x)$ to be the level of 'happiness' for an agent having a final wealth of $x$ units (at time $T$ ) as valued at time 0 by the (state-complete) market. See the discussion after the definitions of the problems of optimization in Section 4. This is contrary to the traditional approach where an agent has preferences on their consumption behavior according to their value as discounted by a bank account, and is closer in approach to a utility function that is state dependent. See the literature on state-dependent utilities cited above.

For $s \leq t$, define $\alpha(s, t)=\mathcal{X}\left(s, \mathcal{X}^{-1}(t, \cdot)\right)$. Then $\alpha(s, t)=\alpha\left(s, t^{\prime}\right) \circ \alpha\left(t^{\prime}, t\right)$ for all $s, t$, and $t^{\prime}$ in $[0, T]$. We also observe that if $\alpha^{I}(s, t):=I_{1}\left(s, \mathcal{X}^{-1}(t, \cdot)\right)$ then $\alpha^{I}(s, t) \circ \alpha(t, s)=$ $\alpha^{I}(s, s)$. Throughout this paper, we will assume that the following condition on the utility structure holds. 
Condition 1. (Homogeneity.) Let $\left(U_{1}, U_{2}\right)$ be a state preference structure defined as above. For all $s, t \in[0, T]$, there exist constants $\alpha_{s, t}$ and $\alpha_{s}^{I}$ such that $\alpha(s, t)(x)=\alpha_{s, t} x$ and $\alpha^{I}(s, s)(x)=\alpha_{s}^{I} x$, where $\alpha(s, t)$ and $\alpha^{I}(s, t)$ are defined as above. In this case we say that $\left(U_{1}, U_{2}\right)$ is a homogeneous state preference structure.

A way to see this is to say that the structure for the utility preferences remains the same as time evolves. Next we describe some important examples that satisfy Condition 1.

Example 1. Consider a continuous positive function $h:[0, T] \rightarrow(0, \infty)$, and assume that $U_{1}(t, x)=x^{\alpha} h(t)$ and $U_{2}(x)=c x^{\alpha}$ with $\alpha \in(0,1)$ and $c \geq 0$. This is a state preference structure that satisfies Condition 1. Indeed, in this case,

$$
\alpha_{s, t}=\frac{c^{1 /(1-\alpha)}+\int_{s}^{T} h^{1 /(1-\alpha)}\left(t^{\prime}\right) \mathrm{d} t^{\prime}}{c^{1 /(1-\alpha)}+\int_{t}^{T} h^{1 /(1-\alpha)}\left(t^{\prime}\right) \mathrm{d} t^{\prime}}, \quad \alpha_{t}^{I}=\frac{h^{1 /(1-\alpha)}(t)}{c^{1 /(1-\alpha)}+\int_{t}^{T} h^{1 /(1-\alpha)}\left(t^{\prime}\right) \mathrm{d} t^{\prime}} .
$$

Example 2. Consider a continuous positive function $h$ as above, and assume that $U_{1}(t, x)=$ $h(t) \log (x)$ and $U_{2}(x)=c \log (x)$ with $c \geq 0$. It follows that this is a state preference structure that satisfies Condition 1 with

$$
\alpha_{s, t}=\frac{c+\int_{s}^{T} h\left(t^{\prime}\right) \mathrm{d} t^{\prime}}{c+\int_{t}^{T} h\left(t^{\prime}\right) \mathrm{d} t^{\prime}}, \quad \alpha_{t}^{I}=\frac{h(t)}{c+\int_{t}^{T} h\left(t^{\prime}\right) \mathrm{d} t^{\prime}} .
$$

Example 3. Let $U_{1}(t, x)=h(t) u(x / h(t))$ and $U_{2}(x)=c u(x / c)$, where $u(\cdot)$ is a utility function, $h(\cdot)$ is a positive continuous function, and $c>0$. It follows that $\left(U_{1}, U_{2}\right)$ is a state preference structure that satisfies Condition 1. In this case,

$$
\alpha_{s, t}=\frac{c+\int_{s}^{T} h\left(t^{\prime}\right) \mathrm{d} t^{\prime}}{c+\int_{t}^{T} h\left(t^{\prime}\right) \mathrm{d} t^{\prime}}, \quad \alpha_{t}^{I}=\frac{h(t)}{c+\int_{t}^{T} h\left(t^{\prime}\right) \mathrm{d} t^{\prime}} .
$$

In particular, for any given $c>0, U_{1}(t, x)=u(x)$ and $U_{2}(x)=c u\left(c^{-1} x\right)$ define a state preference structure that satisfies Condition 1.

Let us point out that the coefficients $\alpha_{s, t}$ and $\alpha_{t}^{I}$ for all $s$ and $t$ in Example 3 are independent of the function $u$, and are identical to the coefficients obtained for the similar coefficients of Example 2. In Theorems 2, 3, and 4, below, we show that, for an agent that consumes and invests optimally, as explained in Problems 1, 2, and 3, below, respectively, the values of these variables depend on the utility functions through only the coefficients $\alpha_{s t}$ and $\alpha_{t}$.

\section{A martingale approach}

We remind the reader that we assume throughout this paper that (2) holds, and that $\sigma(s, t, p)$ is a matrix with maximal rank for all $s, t$, and $p$. It follows that if there exists an 'equivalent martingale measure', this measure is unique (see [20]). Therefore, under the assumptions in this paper, if there exists an 'equivalent martingale measure' then $H(0, t, p)$ is the unique stateprice density process. However, the theory developed in [34] and [35] allows us to hedge and to price any reasonable financial instrument even in the case where $\mathrm{E}[Z(s, T, p)]<1$ for some $s$ and $p$. Londoño [34] discussed examples of this kind of model with a clear economic meaning; see also the references therein.

Next, we define a refinement of the concept of the wealth-income structure introduced in [35], which is needed to formulate the optimization problems that we consider. 
Definition 3. Assume that $(X, \Gamma)$ is a wealth-income evolution structure (see [35] for this definition). Assume that $\Gamma \equiv E-C$, where

$$
\mathrm{d} C(s, t, x, p)=c(s, t, x, p) \mathrm{d} t
$$

and

$$
\mathrm{d} E(s, t, p)=\varepsilon(s, t, p) \mathrm{d} t
$$

for nonnegative $(X, P)$-consistent semimartingale processes $c$ and $\varepsilon$ of class $C^{\delta}$ for some $\delta>0$. Moreover, assume that

$$
\mathrm{E}\left[\int_{s}^{T} H(s, u, p) \varepsilon(s, u, p) \mathrm{d} u\right]<\infty
$$

for all $p \in \mathbb{R}_{+}^{n}$ and $0 \leq s \leq T$, where, as before, $H(s, t, p)$ is the state-price density process. Similarly, assume that

$$
\mathrm{E}\left[\int_{s}^{T} H(s, u, p) c(s, u, x, p) \mathrm{d} u\right]<\infty
$$

for all $p \in \mathbb{R}_{+}^{n}, x \in \mathbb{R}$, and $0 \leq s \leq T$.

We say that, as above, $(X, c, \varepsilon)$ is a rate of consumption and endowment evolution structure. We will say that $c$ is the consumption rate evolution structure and that $\varepsilon$ is the endowment rate evolution structure. We also say that $E$ is a cumulative endowment structure and that $C$ is a cumulative consumption structure.

A subsistence random field $L$ is a $P$-consistent process with drift and diffusion of class $C^{\delta}$ for some $\delta>0$, where $L(s, \cdot, p) H(s, \cdot, p)$ is uniformly bounded below for all $p$ and $s$ (where the bound might depend on $p$ and $s$ ) such that

$$
\mathrm{E}[H(s, t, p) L(s, t, p)]<\infty \text { for all } p, s, \text { and } t .
$$

It is natural to believe that the evolution of income due to labor depends only on the evolution of the state of the economy and not on the current wealth of an agent.

Typically, we are interested in consumption and endowment evolution structures whose wealth remains above some given process. Next we present the definition that embodies this idea.

Definition 4. Let $(X, \varepsilon, c)$ be a hedgeable (by a state tame portfolio) rate of consumption and endowment evolution structure, as in Definition 3, with portfolio evolution structure $\left(\pi_{0}, \pi\right)$. We will say that the couple $(\pi, c)$ of portfolio on stocks and rate of consumption is admissible for $(L, \varepsilon)$, where $L$ is a subsistence random field, and we write $(\pi, c) \in \mathcal{A}(L, \varepsilon)$ if, for any $x$, $s$, and $p$ with $x \geq L(s, s, p)$,

$$
X(s, t, x, p) \geq L(s, t, p) \text { for all } t .
$$

If there are no portfolio on stocks and rate of consumption admissible for $(L, \varepsilon)$, we say that the class cited above is empty, and we denote this by $\mathcal{A}(L, \varepsilon)=\varnothing$.

For any hedgeable wealth and income evolution structure $(X, E-C)$ with $(\pi, c)$ admissible for $(L, \varepsilon)$, it must hold that

$$
x \geq \mathrm{E}\left[H(s, T, p) L(s, T, p)+\int_{s}^{T} H(s, u, p)(c(s, u, x, p)-\varepsilon(s, u, p)) \mathrm{d} u\right]
$$


for any $x \geq L(s, s, p)$, where this last assertion follows since the process defined by (7), below (where $L$ is changed to $X$ ), is a supermartingale. It is often the case that $L(s, T, p)=0$ for all $s$ and $p$. In this latter case, the condition for the previous equation becomes

$$
x \geq \mathrm{E}\left[\int_{s}^{T} H(s, u, p)(c(s, u, x, p)-\varepsilon(s, u, p)) \mathrm{d} u\right] .
$$

Next we explain the problem that we are interested in solving in this paper. We consider a subsistence random field $L$ and an endowment rate evolution structure $\varepsilon$. The control stochastic problem that we propose to solve concerns a small investor that at time 0 has an initial capital $x$, is constrained not to let his wealth fall below a subsistence random field $L(0, \cdot, p)$, has a rate of endowment process, $\varepsilon(0, \cdot, p)$, and has at his disposal portfolio/consumption processes $(\pi, c) \in \mathcal{A}(L, \varepsilon)$. The following proposition is a direct consequence of [35, Theorem 2]; it provides conditions under which $\mathcal{A}(L, \varepsilon) \neq \varnothing$.

Proposition 1. Consider a subsistence random field $L$ and a rate evolution structure $\varepsilon$ as in Definition 4. Assume that

$$
H(s, t, p) L(s, t, p)-\int_{s}^{t} H(s, t, p) \varepsilon(s, t, p) \mathrm{d} u
$$

is a martingale for all $s$ and $p$. Then, there exists a rate of consumption and endowment evolution structure $(X, 0, \varepsilon)$ with $(\pi, 0) \in \mathcal{A}(L, \varepsilon)$, where $\pi$ is the portfolio on stocks defined by $X$.

Proof. Define $X$ by

$$
X(s, t, x, p):=L(s, t, p)+(x-L(s, s, p)) H^{-1}(s, t, p) .
$$

It follows using [35, Theorem 2] that $(X, 0, \varepsilon)$ is the desired rate of consumption and endowment evolution structure. This completes the proof.

For the following condition, let $(X, c, \varepsilon)$ be a rate of consumption and endowment evolution structure with discounted payoff process defined as

$$
Y(s, t, x, p):=H(s, t, p) X(s, t, x, p)+\int_{s}^{t} H(s, u, p)(c(s, u, x, p)-\varepsilon(s, u, p)) \mathrm{d} u .
$$

Condition 2. Let $(X, c, \varepsilon)$ be a rate of consumption and endowment evolution structure, as above. We assume that there exist positive constants $\gamma \geq 1$ and $\alpha_{1}, \alpha_{2}, \alpha_{3}, \beta_{0}, \ldots, \beta_{n}$ with $\alpha_{1}^{-1}+\alpha_{2}^{-1}+\alpha_{3}^{-1}+\sum_{i=0}^{n} \beta_{i}<1$ such that the discounted payoff process $Y(s, t, x, p)$ satisfies

$$
\begin{aligned}
& \mathrm{E}\left[\left|Y(x, p, s, t)-Y\left(x^{\prime}, p^{\prime}, s^{\prime}, t^{\prime}\right)\right|^{\gamma}\right] \\
& \quad \leq C\left(\left|s-s^{\prime}\right|^{\alpha_{1}}+\left|t-t^{\prime}\right|^{\alpha_{2}}+\left|x-x^{\prime}\right|^{\alpha_{3}}+\sum_{i=0}^{n}\left|p_{i}-p_{i}^{\prime}\right|^{\beta_{i}}\right) .
\end{aligned}
$$

This condition is usually satisfied when $X$ is a process that solves a stochastic differential equation. For instance, see [31, Lemma 4.5.6]. The above inequality is needed in order to obtain a continuous modification of the random field and its conditional expectation. See Kolmogorov's continuity criterion for random fields [31, Theorem 1.4.1 and Exercise 1.4.12]. 
In this paper, all rates of consumption and endowment evolution structures are assumed to satisfy Condition 2, and conditional expectations of stochastic processes are the continuous modifications of the given stochastic processes.

For the problems of optimal consumption and terminal wealth that we describe below, we will assume that the subsistence random field is defined in such a way that the discounted (by the state-price density process) subsistence random field of an agent cannot fall below the current value of future endowments:

$$
L(s, t, p)=\frac{-1}{H(s, t, p)} \mathrm{E}\left[\int_{t}^{T} H(s, u, p) \varepsilon(s, u, p) \mathrm{d} u \mid \mathcal{F}_{s, t}\right]
$$

for $0 \leq s \leq t \leq T$ and $p \in \mathbb{R}_{+}^{n}$. In fact, it is not difficult to see that the family of stochastic processes defined by the above equation is a subsistence random field with $\mathcal{A}(L, \varepsilon) \neq \varnothing$, since the discounted payoff process $Y(s, t, p)$ satisfies

$$
\begin{aligned}
Y(s, t, p) & =H(s, t, p) L(s, t, p)-\int_{s}^{t} H(s, u, p) \varepsilon(s, u, p) \mathrm{d} u \\
& =-\mathrm{E}\left[\int_{s}^{T} H(s, u, p) \varepsilon(s, u, p) \mathrm{d} u \mid \mathcal{F}_{s, t}\right]
\end{aligned}
$$

and, therefore, is clearly a martingale.

In fact, Proposition 1 is a consequence of a more general theorem stated below. It allows us to solve the problem of optimal consumption and investment under more general subsistence random fields.

Theorem 1. Let $(X, c, \varepsilon)$ be a rate of consumption and endowment evolution structure, as in Definition 3, with cumulative endowment and consumption structures $C$ and $E$, as defined by (5) and (6), respectively (satisfying Condition 2). If the family of processes defined by (7) are martingales for each $x, p$, and s, then $(X, E-C)$ is a hedgeable wealth-income structure.

Proof. This is a straightforward consequence of Theorem 2 and Theorem 3 of [35].

\section{Consumption and portfolio optimization}

In this paper we are interested in solving the optimization problems presented below. We consider a state preference structure $\left(U_{1}, U_{2}\right)$ and a subsistence random field $L$, defined as in (8), with an endowment rate evolution structure $\varepsilon$.

Problem 1. (Utility from consumption.) Under the hypotheses assumed here, the problem of maximizing the expected utility from the discounted consumption (by the state-price density) is defined to be the problem of maximizing

$$
V_{1}(x, p):=\sup _{(\pi, c) \in \mathcal{A}_{1}(L, \varepsilon, x)} \mathrm{E}\left[\int_{0}^{T} U_{1}(t, H(0, t, p) c(0, t, x, p)) \mathrm{d} t\right]
$$

for all $p$ and $x>-\mathrm{E}\left[\int_{0}^{T} H(0, u, p) \varepsilon(0, u, p) \mathrm{d} u\right]$, where

$$
\mathcal{A}_{1}(L, \varepsilon, x):=\left\{(\pi, c) \in \mathcal{A}(L, \varepsilon): \mathrm{E}\left[\int_{0}^{T} U_{1}^{-}(t, H(0, t, p) c(0, t, x, p)) \mathrm{d} t\right]<\infty\right\}
$$

and $U_{1}^{-}(t, x)=-\left(U_{1}(t, x) \wedge 0\right)$. We will say that $V_{1}$ is the value function for this problem. 
Problem 2. (Utility from terminal wealth.) Under the hypotheses assumed in this section, the problem of maximizing the expected utility from the discounted terminal wealth is defined to be the problem of maximizing

$$
V_{2}(x, p):=\sup _{(\pi, c) \in \mathcal{A}_{2}(L, \varepsilon, x)} \mathrm{E}\left[U_{2}(H(0, T, p) X(0, T, x, p))\right]
$$

for all $p$ and $x>-\mathrm{E}\left[\int_{0}^{T} H(0, u, p) \varepsilon(0, u, p) \mathrm{d} u\right]$, where

$$
\mathcal{A}_{2}(L, \varepsilon, x):=\left\{(\pi, c) \in \mathcal{A}(L, \varepsilon): \mathrm{E}\left[U_{2}^{-}(H(0, T, p) X(0, T, x, p))\right]<\infty\right\}
$$

and $U_{2}^{-}(x)=-\left(U_{2}(x) \wedge 0\right)$. We will say that $V_{2}$ is the value function for this problem.

Problem 3. (Utility from both consumption and terminal wealth.) Under the hypotheses assumed above, the problem of maximizing the expected utility from both the discounted consumption and the discounted terminal wealth is defined to be the problem of maximizing

$$
\begin{aligned}
V(x, p):=\sup _{(\pi, c, x) \in \mathcal{A}(L, \varepsilon, x)} \mathrm{E} & {\left[\int_{0}^{T} U_{1}(t, H(0, t, p) c(0, t, x, p)) \mathrm{d} t\right.} \\
& \left.+U_{2}(H(0, T, p) X(0, T, x, p))\right]
\end{aligned}
$$

for all $p$ and $x>-\mathrm{E}\left[\int_{0}^{T} H(0, u, p) \varepsilon(0, u, p) \mathrm{d} u\right]$, where

$$
\mathcal{A}(L, \varepsilon, x):=\mathcal{A}_{1}(L, \varepsilon, x) \cap \mathcal{A}_{2}(L, \varepsilon, x) .
$$

We say that $V$ is the value function for this problem.

A few words are needed here. Using the valuation theory in [35], if $X \in \mathcal{W}(\mathcal{M})$ is a wealth evolution structure with the property that $H(s, t, x, p) X(s, t, x, p)$ is a process that is uniformly bounded below (where the bound might depend on $x, p$, and $s$ ) and such that

$$
x=\mathrm{E}[H(s, T, p) X(s, T, x, p)]<\infty,
$$

then $\mathrm{E}[H(0, T, x, p) X(0, T, x, p)]$ is the value at 0 of $X$, in the sense that it is possible to find a portfolio that replicates $X$ (see the definition of a hedgeable wealth income structure). Let us note that, since $H(s, T, p) X(s, T, x, p)$ is a supermartingale for all $s, x$, and $p$, under the assumption of integrability and boundedness (from below) of this process, then $x \geq \mathrm{E}[H(s, T, p) X(s, T, x, p)]$. Therefore, in order to consider the optimization problem, Problem 2, it is sufficient to look at the wealth processes that satisfy (9). It follows that $H$ is the natural discount process that discounts the value of wealth at any given time to its present value. As a consequence, if the technical conditions of Problem 2 are satisfied, an agent that solves this problem is using the utility as a way to value, at time 0 , his degree of 'happiness' of holding (a final wealth) in the future. Note that 0 is the time when the investment and consumption decisions are being made. Similar remarks hold for the other optimization problems.

We also point out that 0 does not play any special role, and concepts such as wealth, cumulative income, portfolio process, state preference structure, value functions, and alike can be carried out for any time interval $[s, T]$ with $0 \leq s \leq T$. The above remark allows us to consider parameterized utility preference structures with parameter $0 \leq s \leq T$, defined on the time interval $[s, T]$. This models how an agent can change preferences as time evolves. 
The problems considered above are different from the standard problems of optimal consumption and investment; see, for instance, [26, pp. 88-158]. First, the optimization problems are over portfolio and consumptions which are consistent. Second, the utility functions reflect the level of satisfaction over levels of consumption in Problem 1, final wealths in Problem 2, and on both in Problem 3, as valued by the market when the agent is making his consumption and investment decisions (at time 0 ).

Let us define

$$
\Pi(s, t, p):=-\mathrm{E}\left[\int_{t}^{T} H(s, u, p) \varepsilon(s, u, p) \mathrm{d} u \mid \mathcal{F}_{s, t}\right] .
$$

For any $x>\Pi(t, t, p)$, we define $\mathcal{y}(t, x, p)$ as the unique solution of

$$
\mathcal{X}(t, \mathcal{y}(t, x, p))=x-\Pi(t, t, p),
$$

where $X$ is defined by (4). It follows that $y(t, x, p)=X^{-1}(t, x-\Pi(t, t, p))$. Also, for the following theorem, we introduce a function $G$ defined as

$$
G(s, y)=\int_{s}^{T} U_{1}\left(t, I_{1}(t, y)\right) \mathrm{d} t+U_{2}\left(I_{2}(y)\right)
$$

for $0<y<\infty$ and $0 \leq s \leq T$.

Theorem 2. Assume that the hypotheses of Problem 3 hold, and, in addition, assume that $\left(U_{1}, U_{2}\right)$ is a homogeneous state preference structure (see Condition 1). Define $\xi$ as

$$
\xi(s, t, x, p):= \begin{cases}H^{-1}(s, t, p)(\Pi(s, t, p)+\mathcal{X}(t, y(s, x, p))) & \text { if } x>\Pi(s, s, p), \\ H^{-1}(s, t, p)(\Pi(s, t, p)+x-\Pi(s, s, p)) & \text { otherwise, }\end{cases}
$$

and let $c$ be defined as

$$
c(s, t, x, p):= \begin{cases}H^{-1}(s, t, p) I_{1}(t, y(s, x, p)) & \text { if } x>\Pi(s, s, p), \\ 0 & \text { otherwise. }\end{cases}
$$

Then, $(\xi, c, \varepsilon)$ is a hedgeable cumulative consumption and endowment structure with portfolio $(\pi, c) \in \mathcal{A}(L, \varepsilon)$ that is optimal for the problem of optimal consumption and investment. The corresponding optimal portfolio on stocks is

$$
\begin{aligned}
& {\left[\xi(s, t, x, p)-\tilde{\Pi}(t, P(s, t, p))-\phi_{0}(t, P(s, t, p))\right]\left(\sigma \sigma^{\top}\right)^{-1}\left(b+\delta-r \mathbf{1}_{n}\right)(s, t, p)} \\
& -\left(\phi_{1}(t, P(s, t, p)), \ldots, \phi_{n}(t, P(s, t, p))\right)^{\top}
\end{aligned}
$$

where

$$
\tilde{\Pi}(t, p):=\Pi(t, t, p) \quad \text { and } \quad \phi_{i}(t, p):=p_{i} \frac{\partial \Pi(t, t, p)}{\partial p_{i}}, \quad 0 \leq i \leq n .
$$

Proof. Let us point out that Condition 1 implies that $\xi$ is a (consistent) process, and, clearly, it is Lipschitz continuous. The homogeneity also implies that $c$ is a $(\xi, P)$-consistent process 
of class $C^{0,1}$. We observe that

$$
\begin{aligned}
Y(s, t, x, p):= & H(s, t, p) \xi(s, t, x, p)+\int_{s}^{t} H(s, u, p)(c(s, u, x, p)-\varepsilon(s, u, p)) \mathrm{d} u \\
= & x+\mathrm{E} \int_{s}^{T} H(s, u, p) \varepsilon(s, u, p) \mathrm{d} u \\
& -\mathrm{E}\left[\int_{s}^{T} H(s, u, p) \varepsilon(s, u, p) \mathrm{d} u \mid \mathcal{F}_{s, t}\right]
\end{aligned}
$$

is a martingale, and, therefore, Theorem 1 implies that $(\xi, c, \varepsilon)$ is a rate of consumption and endowment structure with portfolio $(\pi, c) \in \mathcal{A}(L, \varepsilon)$. Next, we observe that, for $x>$ $\Pi(s, s, p)$,

$$
\begin{aligned}
& \mathrm{E}\left[\int_{s}^{T} U_{1}(t, H(s, t, p) c(s, t, x, p)) \mathrm{d} t\right]+\mathrm{E}\left[U_{2}(H(s, T, p) \xi(s, T, x, p))\right] \\
& \quad=G(s, y(s, x, p))
\end{aligned}
$$

where $G$ is the function defined in (10). If $\left(X^{\prime}, \epsilon, c^{\prime}\right)$ is a hedgeable rate of consumption, endowment, and wealth evolution structure, then, for $x>\Pi(s, s, p)$,

$$
\begin{aligned}
& \mathrm{E}\left[\int_{s}^{T} U_{1}\left(t, H(s, t, p) c^{\prime}(s, t, x, p)\right) \mathrm{d} t+U_{2}\left(H(s, T, p) X^{\prime}(s, T, x, p)\right)\right] \\
& \quad \leq G(s, y(s, x, p))-y(s, x, p)\left(\int_{s}^{T} I_{1}(t, y(s, x, p)) \mathrm{d} t+I_{2}(y(s, x, p))\right) \\
& \quad+\mathcal{Y}(s, x, p) \mathrm{E}\left[H(s, T, p) X^{\prime}(s, T, x, p)+\int_{s}^{T} H(s, u, p) c^{\prime}(s, u, x, p) \mathrm{d} u\right] \\
& \quad \leq G(s, y(s, x, p)),
\end{aligned}
$$

where the first inequality is a consequence of (3) and the last inequality is a consequence of the fact that the process defined by (7) is a supermartingale for any hedgeable wealth-income structure.

Next we prove that the optimal portfolio satisfies (11). It is known that the corresponding optimal portfolio should satisfy

$$
\sigma^{\top}(s, t, p) \pi(s, t, x, p)=H^{-1}(s, t, p) \varphi(s, t, x, p)+\xi(s, t, x, p) \theta(s, t, p),
$$

where $\varphi(s, t, x, p)$ is the process such that

$$
Y(s, t, x, p)=x+\int_{s}^{t} \varphi^{\top}(s, u, x, p) \mathrm{d} W_{s}(u)
$$

and $Y$ is the discounted (by the state-price density) payoff process defined by (7). Using the uniqueness of the decomposition of a continuous semimartingale as a local martingale and a process of bounded variation, Itô's rule, and the fact that $\varepsilon$ is a $P$-consistent process, it follows by a straightforward computation that the optimal portfolio is given by (11).

Remark 1. If $\varepsilon$ is a $P$-consistent process where $\mathrm{E}\left[\int_{s}^{T} H(s, u, p) \varepsilon(s, u, p) \mathrm{d} u \mid \mathcal{F}_{s, t}\right]$ is a deterministic function then the proof of the above theorem shows that the optimal portfolio is

$$
\pi(s, t, x, p)=\left(\sigma \sigma^{\top}\right)^{-1}\left(b+\delta-r \mathbf{1}_{n}\right)(s, t, p) \xi(s, t, x, p) .
$$


One important example of the above case is when there is no additional income to invest in the portfolio.

Remark 2. One of the consequences of Theorem 2 is that the solution to Problem 3, under the hypothesis that the state preference structure is homogeneous, is also homogeneous in the sense that we explain next. For any time $0 \leq s \leq T$, the solution $(\xi, c)$ of Problem 3 (as well as its associated optimal portfolio) satisfies the property that its restriction to the time interval $[s, T]$ is also optimal for the problem of optimal consumption and investment after time $s$ (where the definition of the solution to the problem has been outlined after the definition of the solution to the problems of optimal consumption and investment). The latter remark is a consequence of the proof of Theorem 2 .

Remark 3. If

$$
\xi_{\varepsilon}(s, t, x, p)=H^{-1}(s, t, p)(\Pi(s, t, p)+x-\Pi(s, s, p))
$$

then $\left(\xi_{\varepsilon}, 0\right)$ is admissible for $(L, \varepsilon)$ and it can be seen as an admissible wealth process obtained from initially having $x$ dollars and the income process derived from the salary $\varepsilon$. It follows that

$$
\xi(s, t, x, p)=\xi_{\varepsilon}(s, t, \Pi(s, s, p) \wedge x, p)+\alpha_{t s} H^{-1}(s, t, p)(x-\Pi(s, s, p))^{+},
$$

where $x^{+}=0 \vee x$ is the positive part of a number. It also follows that

$$
\begin{aligned}
c(s, t, x, p) & =H^{-1}(s, t, p) \frac{\alpha_{t}^{I}}{\alpha_{s t}}(x-\Pi(s, s, p))^{+} \\
& =\frac{\alpha_{t}^{I}}{\alpha_{s t}^{2}\left(\xi(x, t, x, p)-\xi_{\varepsilon}(s, t, \Pi(s, s, p) \wedge x, p)\right)} .
\end{aligned}
$$

These conclusions can be interpreted as follows. If an agent has a homogeneous state preference structure, and the agent acts to maximize the expected utility from the discounted consumption and discounted terminal wealth, as explained in Problem 3, he creates two accounts. In the first account (which we call the regulatory account) he hedges the risk of maintaining the total wealth above the minimal required by society. He puts all the money derived from his salary into this account. In the other account (the consuming account) he invests in a way that its current value (in the future) is a proportion of the initial value. He also consumes for a period of time a proportion of the wealth in his consuming account, and this factor (of proportion) is $\alpha_{t}^{I} / \alpha_{s t}^{2}$.

Next, we present without proof the solution to the problem of maximizing the expected utility from the discounted consumption. The proof is similar to the proof of Theorem 2, and is left to the reader. In order to state the following theorem, we introduce

$$
\chi_{1}(t, y)=\int_{t}^{T} I_{1}\left(t^{\prime}, y\right) \mathrm{d} t^{\prime}
$$

and

$$
G_{1}(t, y)=\int_{t}^{T} U_{1}\left(t^{\prime}, I_{1}\left(t^{\prime}, y\right)\right) \mathrm{d} t^{\prime}
$$

for $y>0$ and $0 \leq t \leq T$. We also set $\mathcal{Y}_{1}(t, x, p)=\mathcal{X}_{1}^{-1}(t, x-\Pi(t, t, p))$ for $x>0$ and $0 \leq t \leq T$. 
Theorem 3. Consider a homogeneous state preference structure $\left(U_{1}, U_{2}\right)$ and subsistence random field $L$, defined as in (8), with an endowment rate evolution structure $\varepsilon$. Define $\xi_{1}$ as

$$
\xi_{1}(s, t, x, p):= \begin{cases}H^{-1}(s, t, p)\left(\Pi(s, t, p)+\chi_{1}\left(t, \mathcal{Y}_{1}(s, x, p)\right)\right) & \text { if } x>\Pi(s, s, p), \\ H^{-1}(s, t, p)(\Pi(s, t, p)+x-\Pi(s, s, p)) & \text { otherwise, }\end{cases}
$$

and let $c_{1}$ be defined as

$$
c_{1}(s, t, x, p):= \begin{cases}H^{-1}(s, t, p) I_{1}\left(t, \mathcal{Y}_{1}(s, x, p)\right) & \text { if } x>\Pi(s, s, p), \\ 0 & \text { otherwise. }\end{cases}
$$

Then, $\left(\xi_{1}, c_{1}, \varepsilon\right)$ is a rate of consumption and endowment structure with portfolio $\left(\pi_{1}, c_{1}\right) \in$ $\mathcal{A}(L, \varepsilon)$ that is optimal for Problem 1. The optimal portfolio on stocks is

$$
\begin{aligned}
& {\left[\xi_{1}(s, t, x, p)-\tilde{\Pi}(t, P(s, t, p))-\phi_{0}(t, P(s, t, p))\right]\left(\sigma \sigma^{\top}\right)^{-1}\left(b+\delta-r \mathbf{1}_{n}\right)(s, t, p)} \\
& -\left(\phi_{1}(t, P(s, t, p)), \ldots, \phi_{n}(t, P(s, t, p))\right)^{\top},
\end{aligned}
$$

where $\Pi$ and $\phi_{i}, 0 \leq i \leq n$, are defined as in Theorem 2 .

Also, the solution for problem (8) is stated without a proof.

Theorem 4. Consider a homogeneous state preference structure and subsistence random field $L$, defined as in (8), with an endowment rate evolution structure $\varepsilon$. Let $\xi_{2}$ be defined as

$$
\xi_{2}(s, t, x, p):=H^{-1}(s, t, p)(\Pi(s, t, p)+x-\Pi(s, s, p)) .
$$

Then, $\left(\xi_{2}, 0, \varepsilon\right)$ is a rate of consumption and endowment structure with portfolio $\left(\pi_{2}, 0\right) \in$ $\mathcal{A}(L, \varepsilon)$ that is optimal for Problem 2. The optimal portfolio on stocks is

$$
\begin{aligned}
& {\left[\xi_{2}(s, t, x, p)-\tilde{\Pi}(t, P(s, t, p))-\phi_{0}(t, P(s, t, p))\right]\left(\sigma \sigma^{\top}\right)^{-1}\left(b+\delta-r \mathbf{1}_{n}\right)(s, t, p)} \\
& -\left(\phi_{1}(t, P(s, t, p)), \ldots, \phi_{n}(t, P(s, t, p))\right)^{\top},
\end{aligned}
$$

where $\Pi$ and $\phi_{i}, 0 \leq i \leq n$, are defined as in Theorem 2 .

Remark 4. We point out that the wealth process and the portfolio process obtained in the solution of Problem 2 are independent of the utility function.

\section{Acknowledgements}

I want to thank participants of the fourth world congress of the Bachelier Finance Society in Tokyo where this paper was presented for valuable comments. All remaining errors are mine. This work was supported by COLCIENCIAS (Colombian National Science Foundation) under grant 284-2003, Universidad EAFIT, and Universidad Nacional de Bogotá. I also want to thank an anonymous referee for a detailed reading of this paper and valuable suggestions that led to a substantial improvement of the paper.

\section{References}

[1] Balduzzi, P. AND Lynch, A. (1999). Transaction costs and predictability: some utility cost calculations. J. Financial Econom. 52, 47-78.

[2] Brennan, M. J. (1998). The role of learning in dynamic portfolio decisions. Europ. Finance Rev. 1, $295-306$. 
[3] Brennan, M. J. and Xia, Y. (2001). Dynamic asset allocation under inflation. J. Finance 57, 1201-1238.

[4] Brennan, M. J., Schwartz, E. S. and Lagnado, R. (1997). Strategic asset allocation. J. Econom. Dynam. Control 21, 1377-1403.

[5] Campbell, J. and Viceira, L. (1999). Consumption and portfolio decisions when expected returns are time varying. Quart. J. Econom. 114, 433-495.

[6] Campbell, J. and Viceira, L. (2001). Who should buy long-term bonds? Amer. Econom. Rev. 91, 99-127.

[7] Campbell, J. Y., Chacko, G., Rodriguez, J. and Viceira, L. M. (2004). Strategic asset allocation in a continuous-time VAR model. J. Econom. Dynam. Control 28, 2195-2214.

[8] Chabi-Yo, F., Garcia, R. and Renault, E. (2007). State dependence can explain the risk aversion puzzle. Rev. Financial Studies 21, 973-1011.

[9] Constantinides, G. M. (1990). Habit formation: a resolution of the equity premium puzzle. J. Political Econom. 98, 519-543.

[10] Cox, J. C. AND Huang, C.-F. (1989). Optimal consumption and portfolio policies when asset prices follow a diffusion process. J. Econom. Theory 49, 33-83.

[11] Cvitanić, J., Goukasian, L. and Zapatero, F. (2003). Monte Carlo computation of optimal portfolios in complete markets. J. Econom. Dynam. Control 27, 971-986.

[12] Dammon, R. M., Spatt, C. S. And Zhang, H. H. (2001). Optimal consumption and investment with capital gains taxes. Rev. Financial Studies 14, 583-616.

[13] Dangl, T. AND WiRL, F. (2004). Investment under uncertainty: calculating the value function when the Bellman equation cannot be solved analytically. J. Econom. Dynam. Control 28, 1437-1460.

[14] Davis, M. H. A. And Norman, A. R. (1990). Portfolio selection with transaction costs. Math. Operat. Res. 15, 676-713.

[15] Detemple, J. B., Garcia, R. and Rindisbacher, M. (2003). A Monte Carlo method for optimal portfolios. J. Finance 58, 401-446.

[16] Duffie, D. and Epstein, L. G. (1992). Stochastic differential utility. Econometrica 60, 353-394.

[17] Epstein, L. G. AND ZIN, S. E. (1989). Substitution, risk aversion, and the temporal behavior of consumption and asset returns: theoretical framework. Econometrica 57, 937-969.

[18] Gordon, S. and St-Amour, P. (2000). A preference regime model of bull and bear markets. Amer. Econom. Rev. 90, 1019-1033.

[19] Gordon, S. And St-Amour, P. (2004). Asset returns and state-dependent risk preferences. J. Business Econom. Statist. 22, 241-252.

[20] Harrison, J. M. and Pliska, S. R. (1983). A stochastic calculus model of continuous trading: complete markets. Stoch. Process. Appl. 15, 313-316.

[21] Hindy, A. And Hunng, C.-F. (1993). Optimal consumption and portfolio rules with durability and local substitution. Econometrica 61, 85-122.

[22] Hindy, A., HuAng, C.-F. AND Zhu, S. H. (1997). Numerical analysis of a free-boundary singular control problem in financial economics. J. Econom. Dynam. Control 21, 297-327.

[23] Hindy, A., Huanga, C.-F. And Zhu, S. H. (1997). Optimal consumption and portfolio rules with durability and habit formation. J. Econom. Dynam. Control 21, 525-550.

[24] IsEN, A. (2004). Handbook of Emotions, 2nd edn. Guilford Press, New York.

[25] Jackwerth, J. C. (2000). Recovering risk aversion from option prices and realized returns. Rev. Financial Studies 13, 433-451.

[26] Karatzas, I. And Shreve, S. E. (1998). Methods of Mathematical Finance (Appl. Math. 39). Springer, New York.

[27] Karatzas, I., Lehoczky, J. P. and Shreve, S. E. (1987). Optimal portfolio and consumption decisions for a 'small investor' on a finite horizon. SIAM J. Control Optimization 25, 1557-1586.

[28] KARNI, E. (1993). A definition of subjective probabilities with state-dependent preferences. Econometrica 61, 187-198.

[29] Karni, E. (1993). Subjective expected utility theory with state-dependent preferences. J. Econom. Theory 60, $428-438$.

[30] Kim, T. S. AND OMberg, E. (1996). Dynamic nonmyopic portfolio behavior. Rev. Financial Studies 9, 141-161.

[31] Kunita, H. (1990). Stochastic Flows and Stochastic Differential Equations (Camb. Stud. Adv. Math. 24). Cambridge University Press.

[32] LazraK, A. And Quenez, M. C. (2003). A generalized stochastic differential utility. Math. Operat. Res. 28, $154-180$.

[33] Lioui, A. And Poncet, P. (2001). On optimal portfolio choice under stochastic interest rates. J. Econom. Dynam. Control 25, 1841-1865.

[34] Londoño, J. A. (2004). State tameness: a new approach for credit constrains. Electron. Commun. Prob. 9, 1-13.

[35] Londoño, J. A. (2008). A more general valuation and arbitrage theory for Itô processes. Stoch. Anal. Appl. 26, 809-831. 
[36] Magill, M. J. P. and Constantinides, G. M. (1976). Portfolio selection with transactions costs. J. Econom. Theory 13, 245-263.

[37] Mehra, R. And Prescott, E. (1985). The equity premium: a puzzle. J. Monetary Econom. 15, 145-161.

[38] Melino, A. ANd Yang, A. X. (2003). State dependent preferences can explain the equity premium puzzle. Rev. Econom. Dynam. 6, 806-830.

[39] Merton, R. C. (1969). Lifetime portfolio selection under uncertainty: the continuous time case. Rev. Econom. Statist. 51, 247-257.

[40] Merton, R. C. (1971). Optimum consumption and portfolio rules in a continuous-time model. J. Econom. Theory 3, 373-413.

[41] Ocone, D. L. And Karatzas, I. (1991). A generalized Clark representation formula, with application to optimal portfolios. Stoch. Stoch. Reports 34, 187-220.

[42] Shreve, S. E. And Soner, H. M. (1994). Optimal investment and consumption with transaction costs. Ann. Appl. Prob. 4, 609-692.

[43] Watchter, J. (2002). Portfolio and consumption decisions under mean-reverting returns: an exact solution for complete markets. J. Financial Quant. Anal. 37, 63-91.

[44] WeIL, O. (1989). The equity premium puzzle and the risk-free rate puzzle. J. Monetary Econom. 24, 401-421. 